\section{E REPORTS}

ISSN $1600-5368$

\section{Crystal structure of bis $\{N$-[2-(dimethyl- amino)ethyl]quinolin-8-amine- $\left.\kappa^{3} N, N^{\prime}, N^{\prime \prime}\right\}$ nickel(II) dichloride \\ 3.5-hydrate}

\author{
Benson M. Kariuki ${ }^{\mathrm{a}}$ and Abdul-Razak H. Al-Sudani ${ }^{\mathrm{b}} *$ \\ ${ }^{a}$ School of Chemistry, Cardiff University, Main Building, Park Place, Cardiff \\ CF10 3AT, Wales, and ${ }^{\mathbf{b}}$ Department of Chemistry, College of Science, Baghdad \\ University for Women, Baghdad, Iraq. *Correspondence e-mail: \\ alsudani@uobaghdad.edu.iq
}

Received 10 August 2014; accepted 22 August 2014

Edited by G. Smith, Queensland University of Technology, Australia

In the title compound, $\left[\mathrm{Ni}\left(\mathrm{C}_{13} \mathrm{H}_{17} \mathrm{~N}_{3}\right)_{2}\right] \mathrm{Cl}_{2} \cdot 3.5 \mathrm{H}_{2} \mathrm{O}$, the geometry of the $\mathrm{NiN}_{6}$ complex cation is slightly distorted octahedral, with a facial arrangement of the two tridentate $\mathrm{N}$-[2(dimethylamino)ethyl]quinolin-8-amine ligands around the metal ion. The asymmetric unit consists of two independent complex half-molecules located on centres of inversion, together with two chloride counter-anions and 3.5 water molecules of solvation, one of which is disordered across an inversion centre. In the crystal, $\mathrm{O}-\mathrm{H} \cdots \mathrm{O}, \mathrm{O}-\mathrm{H} \cdots \mathrm{Cl}$ and $\mathrm{N}-\mathrm{H} \cdots \mathrm{Cl}$ hydrogen-bonding interactions form a threedimensional network structure.

Keywords: crystal structure; $\mathrm{N}$-[2-(dimethylamino)ethyl]quinolin-8-amine; nickel(II) complex; hydrogen bonding.

CCDC reference: 1020674

\section{Related literature}

For background to $\mathrm{N}$-containing ligands, including quinoline derivatives, see: Kizirian (2008); Miodragovic et al. (2008); Puviarasan et al. (2004); Singh et al. (2008); Zhang et al. (2009). For complexes incorporating $N$-[2-(dimethylamino)ethyl]quinolin-8-amine, see: Al-Sudani \& Kariuki (2013); Al-Sudani (2014).

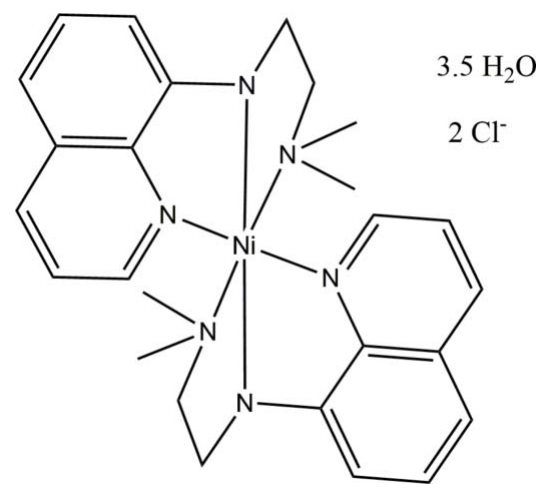

2. Experimental

2.1. Crystal data

$\left[\mathrm{Ni}\left(\mathrm{C}_{13} \mathrm{H}_{17} \mathrm{~N}_{3}\right)_{2}\right] \mathrm{Cl}_{2} \cdot 3.5 \mathrm{H}_{2} \mathrm{O}$

$M_{r}=623.26$

Triclinic, $P \overline{1}$

$a=10.6940$ (2) ^

$b=11.8612$ (4) $\AA$

$c=12.1088(3) \AA$

$\alpha=90.520(1)^{\circ}$

$\beta=101.181$ (2)

$\gamma=102.259(2)^{\circ}$

$V=1470.39(7) \AA^{3}$

$Z=2$

Mo $K \alpha$ radiation

$\mu=0.88 \mathrm{~mm}^{-1}$

$T=150 \mathrm{~K}$

$0.18 \times 0.16 \times 0.08 \mathrm{~mm}$

\subsection{Data collection}

Nonius KappaCCD diffractometer Absorption correction: multi-scan (DENZO/SCALEPACK;

Otwinowski \& Minor, 1997)

$T_{\min }=0.857, T_{\max }=0.933$

10258 measured reflections 6714 independent reflections 5592 reflections with $I>2 \sigma(I)$ $R_{\text {int }}=0.023$

\subsection{Refinement}

$R\left[F^{2}>2 \sigma\left(F^{2}\right)\right]=0.039$

$w R\left(F^{2}\right)=0.090$

$S=1.03$

6714 reflections

383 parameters

12 restraints

$\mathrm{H}$ atoms treated by a mixture of independent and constrained refinement

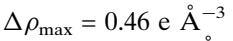

$\Delta \rho_{\min }=-0.59$ e $\AA^{-3}$

Table 1

Hydrogen-bond geometry $\left(\AA{ }^{\circ}\right)$.

\begin{tabular}{|c|c|c|c|c|}
\hline$D-\mathrm{H} \cdots A$ & $D-\mathrm{H}$ & $\mathrm{H} \cdots A$ & $D \cdots A$ & $D-\mathrm{H} \cdots A$ \\
\hline $\mathrm{N} 2-\mathrm{H} 2 A \cdots \mathrm{Cl} 2$ & 1.00 & 2.27 & $3.2038(18)$ & 155 \\
\hline 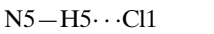 & 1.00 & 2.33 & 3.2715 (19) & 156 \\
\hline $\mathrm{O} 1-\mathrm{H} 1 O 1 \cdots \mathrm{O} 2^{\mathrm{i}}$ & $0.83(1)$ & $1.71(1)$ & $2.541(5)$ & $173(6)$ \\
\hline $\mathrm{O} 1-\mathrm{H} 2 O 1 \cdots \mathrm{O} 2^{\mathrm{ii}}$ & $0.84(1)$ & $2.05(2)$ & $2.863(5)$ & $165(6)$ \\
\hline $\mathrm{O} 2-\mathrm{H} 1 O 2 \cdots \mathrm{Cl}^{\mathrm{iii}}$ & $0.84(1)$ & $2.33(1)$ & $3.162(3)$ & $172(4)$ \\
\hline $\mathrm{O} 2-\mathrm{H} 2 \mathrm{O} 2 \cdots \mathrm{Cl}^{\mathrm{iv}}$ & $0.83(1)$ & $2.37(2)$ & $3.179(2)$ & $163(4)$ \\
\hline $\mathrm{O} 3-\mathrm{H} 1 \mathrm{O}_{3} \cdots \mathrm{Cl}_{2}^{\mathrm{v}}$ & $0.85(1)$ & $2.38(1)$ & $3.222(2)$ & $174(3)$ \\
\hline $\mathrm{O} 3-\mathrm{H} 2 \mathrm{O} 3 \cdots \mathrm{Cl}^{\mathrm{v}}$ & $0.85(1)$ & $2.36(1)$ & 3.2008 (19) & $170(3)$ \\
\hline $\mathrm{O} 4-\mathrm{H} 1 O 4 \cdots \mathrm{O}^{\mathrm{vi}}$ & $0.85(1)$ & $1.91(1)$ & $2.755(5)$ & $177(4)$ \\
\hline $\mathrm{O} 4-\mathrm{H} 2 \mathrm{O} 4 \cdots \mathrm{Cl} 1$ & $0.85(1)$ & $2.33(1)$ & $3.180(3)$ & $173(3)$ \\
\hline
\end{tabular}

Symmetry codes: (i) $x, y-1, z$; (ii) $-x,-y+1,-z+2$; (iii) $-x,-y+1,-z+1$; (iv)

$x, y, z+1 ;(\mathrm{v})-x+1,-y+1,-z+1 ;$ (vi) $x, y+1, z-1$.

Data collection: COLLECT (Nonius, 2000); cell refinement: SCALEPACK (Otwinowski \& Minor, 1997); data reduction: DENZO (Otwinowski \& Minor, 1997) and SCALEPACK; 
program(s) used to solve structure: SIR92 (Altomare et al., 1993); program(s) used to refine structure: SHELXL2013 (Sheldrick, 2008); molecular graphics: Mercury (Macrae et al., 2008), ORTEP-3 for Windows (Farrugia, 2012) and CHEMDRAW Ultra (Cambridge Soft, 2001); software used to prepare material for publication: publCIF (Westrip, 2010).

\section{Acknowledgements}

Gratitude is expressed to Professor P. G. Edwards of the School of Chemistry, Cardiff University, for the opportunity for ARHAS to work in his laboratory as an academic visitor for many years, without which this work would not have been accomplished, as well as for his invaluable advice and financial support.

Supporting information for this paper is available from the $\mathrm{IUCr}$ electronic archives (Reference: ZS2312).

\section{References}

Al-Sudani, A.-R. H. (2014). Acta Cryst. E70, m1.

Al-Sudani, A.-R. H. \& Kariuki, B. M. (2013). Acta Cryst. E69, m491-m492.

Altomare, A., Cascarano, G., Giacovazzo, C. \& Guagliardi, A. (1993). J. Appl. Cryst. 26, 343-350.

Cambridge Soft (2001). CHEMDRAW Ultra. Cambridge Soft Corporation, Cambridge, Massachusetts, USA.

Farrugia, L. J. (2012). J. Appl. Cryst. 45, 849-854.

Kizirian, J.-C. (2008). Chem. Rev. 108, 140-205.

Macrae, C. F., Bruno, I. J., Chisholm, J. A., Edgington, P. R., McCabe, P., Pidcock, E., Rodriguez-Monge, L., Taylor, R., van de Streek, J. \& Wood, P. A. (2008). J. Appl. Cryst. 41, 466-470.

Miodragovic, D. U., Mitic, D. M., Miodragovic, Z. M., Bogdanovic, G. A. \& Vitnik, Z. (2008). Inorg. Chim. Acta, 361, 86-94.

Nonius (2000). COLLECT. Nonius BV, Delft, The Netherlands.

Otwinowski, Z. \& Minor, W. (1997). Methods in Enzymology, Vol. 276, Macromolecular Crystallography, Part A, edited by C. W. Carter Jr \& R. M. Sweet, pp. 307-326. New York: Academic Press.

Puviarasan, N., Arjunan, V. \& Mohan, S. (2004). Turk. J. Chem. 28, 53-65.

Sheldrick, G. M. (2008). Acta Cryst. A64, 112-122.

Singh, A. K., Kumari, S. \& Kumar, K. R. (2008). Polyhedron, 27, 181-186.

Westrip, S. P. (2010). J. Appl. Cryst. 43, 920-925.

Zhang, J.-A., Pan, M., Zhang, J.-Y., Kang, B.-S. \& Su, C.-Y. (2009). Inorg. Chim. Acta, 362, 3519-3525. 


\section{supporting information}

Acta Cryst. (2014). E70, m339-m340 [doi:10.1107/S1600536814019035]

\section{Crystal structure of bis $\{\mathrm{N}$-[2-(dimethylamino)ethyl]quinolin-8-amine- $\boldsymbol{\kappa}^{3} N, N^{\prime}, N^{\prime \prime}$ \}nickel(II) dichloride 3.5-hydrate}

\section{Benson M. Kariuki and Abdul-Razak H. Al-Sudani}

\section{S1. Comment}

As stated previously, (Al-Sudani \& Kariuki, 2013), metal complexes of N-containing ligands occupy an important position in coordination chemistry (Singh et al., 2008; Miodragovic et al., 2008; Zhang et al., 2009). Some quinolinecontaining ligands show interesting biological activities (Puviarasan et al., 2004). 8-[2-(dimethylamino)ethylamino]quinoline ( $\left.\mathrm{NN}^{\prime} \mathrm{N}^{\prime \prime}\right)$, is an asymmetric and potentially tridentate chelating ligand with the same donor atoms. With zinc (Al-Sudani, 2014) and cadmium ions (Al-Sudani \& Kariuki, 2013), it forms neutral 1:1 metal to ligand mole ratio complexes with monomeric distorted square-pyrimidal and dimeric distorted octahedral geometries, respectively. In the nickel(II) complex with the ligand 8-[2-(dimethylamino)ethylamino] quinoline, the title compound, $\mathrm{C}_{26} \mathrm{H}_{34} \mathrm{~N}_{6} \mathrm{Ni}$. $2 \mathrm{Cl} \cdot 3.5\left(\mathrm{H}_{2} \mathrm{O}\right)$, is ionic with a 1:2 metal to ligand mole ratio and has a slightly distorted bis-tridentate $\mathrm{NiN}_{6}$ octahedral coordination [Ni- $\mathrm{N}$ bond length range, 2.0777 (16)-2.2397 (17) $\AA]$ (Fig. 1). The asymmetric unit consists of two independent half molecules with the $\mathrm{Ni}^{2+}$ ions ( $\mathrm{Ni1}$ and $\mathrm{Ni2}$ ) located on centres of inversion, as well as two chloride anions and 3.5 water molecules, one of which $(\mathrm{O} 1)$ is disordered across an inversion centre. A network of $\mathrm{O}-\mathrm{H} \cdots \mathrm{O}, \mathrm{O}-$ $\mathrm{H} \cdots \mathrm{Cl}$ and $\mathrm{N}-\mathrm{H} \cdots \mathrm{Cl}$ hydtrogen-bonding interactions are present in the crystal structure (Table 1) giving a threedimensional network (Fig. 2). In the three complexes with this ligand ( $\mathrm{Zn}, \mathrm{Cd}$ and $\mathrm{Ni}$ ), the unequivelant nitrogen donor atoms of the ligands are arranged facially around the metal ion.

\section{S2. Experimental}

To a stirred methanoic solution $(30 \mathrm{ml})$ containing a slight excess of the ligand (NN'N") $(0.9 \mathrm{~g} ; 0.0042 \mathrm{~mol})$ kept under a positive nitrogen pressure, a methanoic solution $(20 \mathrm{ml})$ of $\mathrm{NiCl}_{2} .6 \mathrm{H}_{2} \mathrm{O}(0.47 \mathrm{~g} ; 0.002 \mathrm{~mol})$ was slowly added. The resulting brownish violet solution was stirred at room temperature overnight. A small amount of anhydrous $\mathrm{MgSO}_{4}$ was added and the reaction solution was stirred for a further one hour. After the removal of the drying agent by filtration, the solvent was removed by vacuum. The solid was washed twice with a small amount of diethyl ether $(15 \mathrm{ml})$ to remove any of the unreacted ligand and was then dried under vacuum at $50{ }^{\circ} \mathrm{C}$. The mass of the collected solid was $0.5 \mathrm{~g}$ which, based on the molecular formula of [ $\left.\mathrm{Ni}\left(\mathrm{NN}^{\prime} \mathrm{N}^{\prime \prime}\right) 2\right] \mathrm{Cl}_{2}$ represented a yield of $c a$. 45\%. A suitable light brown-violet block shaped crystal of the title complex was obtained via slow diffusion of diethyl ether into a small amount of an acetonitrile solution of the compound kept under an atmosphere of nitrogen gas. Single crystal X-ray structure determination has identified the complex as $\left[\mathrm{Ni}\left(\mathrm{NN}^{\prime} \mathrm{N} "\right) 2\right] \mathrm{Cl}_{2} \cdot 3 \cdot 5 \mathrm{H}_{2} \mathrm{O}$. In the process of measuring the melting point, the colour of the crystalline brown-violet solid changed to pale green and finally to very dark green. The dark green material was identified as a bimetallic complex of the formula $\left[\mathrm{Ni}\left(\mathrm{NN}^{\prime} \mathrm{N}^{\prime \prime}\right) \mathrm{Cl}_{2}\right]_{2}$ which decomposed at $c a .240{ }^{\circ} \mathrm{C}$. 


\section{S3. Refinement}

$\mathrm{H}$ atoms were positioned geometrically $(\mathrm{C}-\mathrm{H}=0.95-0.99 \AA$ and $\mathrm{N}-\mathrm{H}=1.00 \AA)$ and refined using a riding model, with $U_{\text {iso }}(\mathrm{H})$ constrained to be 1.2 times $U_{\text {eq }}$ of the bonded atom except for the methyl groups where it was 1.5 times with free rotation about the $\mathrm{C}-\mathrm{C}$ bond. The geometry of the water molecules was constrained during refinement with $\mathrm{O}-\mathrm{H}=$ $0.84(2) \AA ̊$ and $U_{\text {iso }}(\mathrm{H})=1.5$ times $U_{\text {eq }}(\mathrm{O})$.
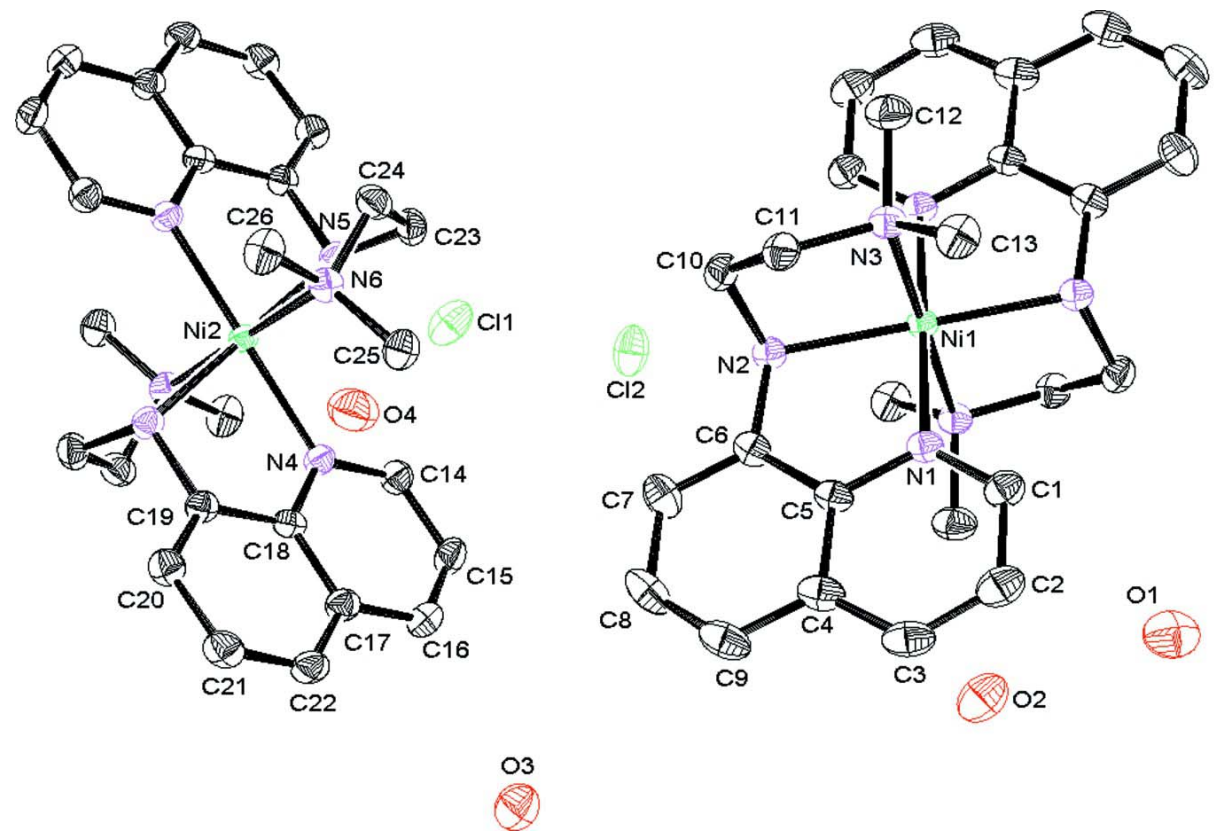

\section{Figure 1}

The asymmetric of the title complex showing atom labels and 50\% probability displacement ellipsoids. Hydrogen atoms have been omitted. 


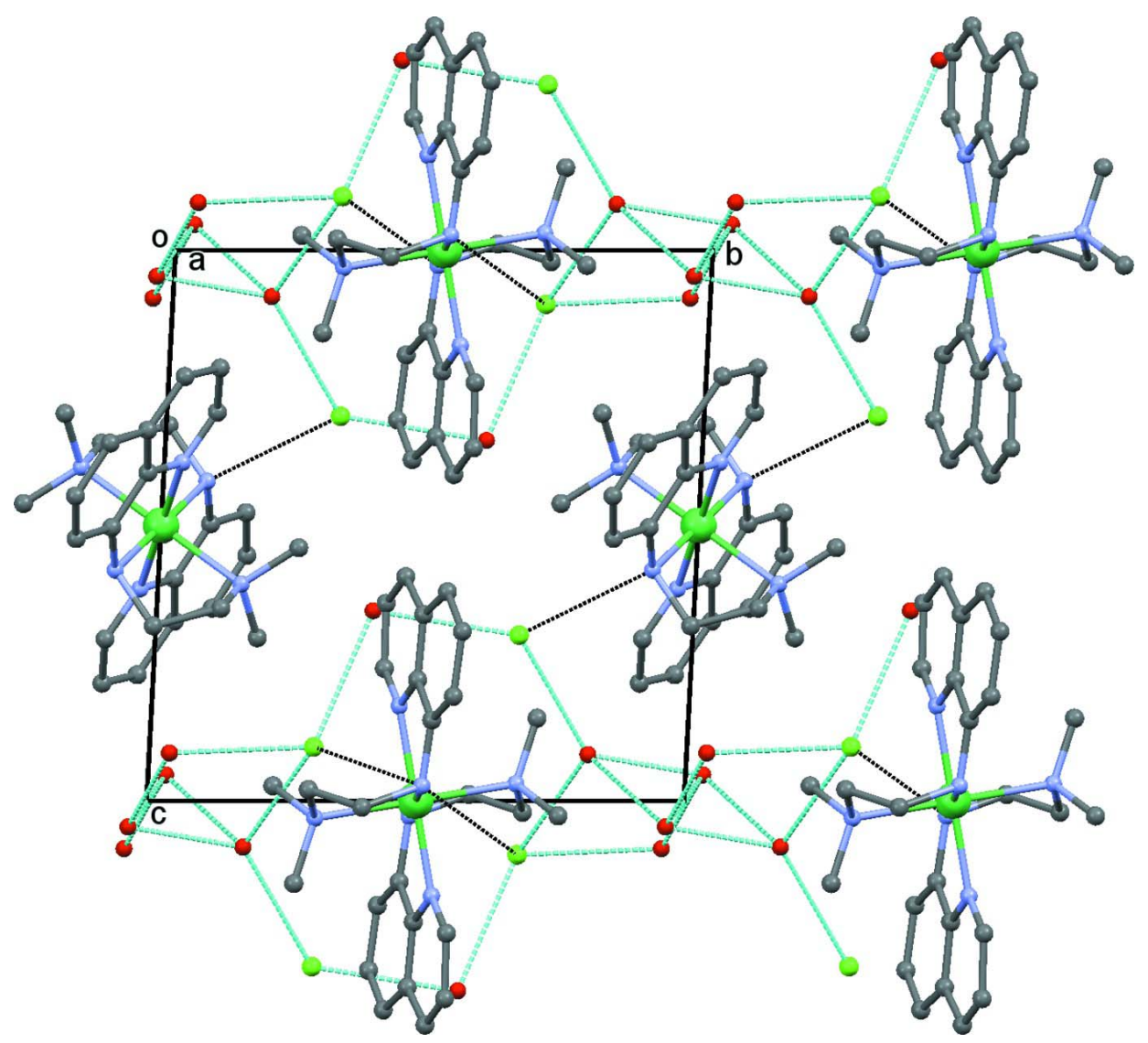

Figure 2

Packing in the crystal structure showing the $\mathrm{O}-\mathrm{H} \cdots \mathrm{O}, \mathrm{O}-\mathrm{H} \cdots \mathrm{Cl}$ and $\mathrm{N}-\mathrm{H} \cdots \mathrm{Cl}$ interactions as dotted lines.

\section{$\operatorname{Bis}\left\{N\right.$-[2-(dimethylamino)ethyl]quinolin-8-amine- $\left.\kappa^{3} N, N^{\prime}, N^{\prime \prime}\right\}$ nickel(II) dichloride 3.5-hydrate}

\section{Crystal data}

$\left[\mathrm{Ni}\left(\mathrm{C}_{13} \mathrm{H}_{17} \mathrm{~N}_{3}\right)_{2}\right] \mathrm{Cl}_{2} \cdot 3.5 \mathrm{H} 2 \mathrm{O}$

$M_{r}=623.26$

Triclinic, $P \overline{1}$

$a=10.6940(2) \AA$

$b=11.8612(4) \AA$

$c=12.1088(3) \AA$

$\alpha=90.520(1)^{\circ}$

$\beta=101.181(2)^{\circ}$

$\gamma=102.259(2)^{\circ}$

$V=1470.39(7) \AA^{3}$

\section{Data collection}

Nonius KappaCCD

diffractometer

Radiation source: fine-focus sealed tube

Graphite monochromator

CCD slices, $\omega$ and $\varphi$ scans
$Z=2$

$F(000)=658$

$D_{\mathrm{x}}=1.408 \mathrm{Mg} \mathrm{m}^{-3}$

Mo $K \alpha$ radiation, $\lambda=0.71073 \AA$

Cell parameters from 5592 reflections

$\theta=2.0-25.2^{\circ}$

$\mu=0.88 \mathrm{~mm}^{-1}$

$T=150 \mathrm{~K}$

Block, violet

$0.18 \times 0.16 \times 0.08 \mathrm{~mm}$ 


\author{
$R_{\text {int }}=0.023$ \\ $\theta_{\max }=27.7^{\circ}, \theta_{\min }=2.0^{\circ}$ \\ $h=-13 \rightarrow 13$

\section{Refinement} \\ Refinement on $F^{2}$ \\ Least-squares matrix: full \\ $R\left[F^{2}>2 \sigma\left(F^{2}\right)\right]=0.039$ \\ $w R\left(F^{2}\right)=0.090$ \\ $S=1.03$ \\ 6714 reflections \\ 383 parameters \\ 12 restraints
}

$k=-14 \rightarrow 15$
$l=-15 \rightarrow 15$

Hydrogen site location: mixed

$\mathrm{H}$ atoms treated by a mixture of independent and constrained refinement

$w=1 /\left[\sigma^{2}\left(F_{\mathrm{o}}^{2}\right)+(0.0261 P)^{2}+1.4643 P\right]$

where $P=\left(F_{\mathrm{o}}^{2}+2 F_{\mathrm{c}}{ }^{2}\right) / 3$

$(\Delta / \sigma)_{\max }<0.001$

$\Delta \rho_{\max }=0.46 \mathrm{e}^{-3}$

$\Delta \rho_{\min }=-0.59$ e $\AA^{-3}$

Special details

Geometry. All e.s.d.'s (except the e.s.d. in the dihedral angle between two 1.s. planes) are estimated using the full covariance matrix. The cell e.s.d.'s are taken into account individually in the estimation of e.s.d.'s in distances, angles and torsion angles; correlations between e.s.d.'s in cell parameters are only used when they are defined by crystal symmetry. An approximate (isotropic) treatment of cell e.s.d.'s is used for estimating e.s.d.'s involving 1.s. planes.

Fractional atomic coordinates and isotropic or equivalent isotropic displacement parameters $\left(\AA^{2}\right)$

\begin{tabular}{|c|c|c|c|c|c|}
\hline & $x$ & $y$ & $z$ & $U_{\text {iso }} * / U_{\text {eq }}$ & Occ. $(<1)$ \\
\hline $\mathrm{C} 1$ & $0.1605(2)$ & $-0.0934(2)$ & $0.70598(18)$ & $0.0262(5)$ & \\
\hline H1 & 0.0799 & -0.1382 & 0.7180 & $0.031 *$ & \\
\hline $\mathrm{C} 2$ & $0.2745(2)$ & $-0.0947(2)$ & 0.78635 (19) & $0.0335(5)$ & \\
\hline $\mathrm{H} 2$ & 0.2703 & -0.1398 & 0.8505 & $0.040^{*}$ & \\
\hline $\mathrm{C} 3$ & $0.3911(2)$ & $-0.0301(2)$ & $0.7709(2)$ & $0.0336(5)$ & \\
\hline $\mathrm{H} 3$ & 0.4685 & -0.0283 & 0.8255 & $0.040 *$ & \\
\hline $\mathrm{C} 4$ & $0.3964(2)$ & $0.0340(2)$ & $0.67356(19)$ & $0.0277(5)$ & \\
\hline $\mathrm{C} 5$ & $0.27726(19)$ & $0.02971(18)$ & $0.59635(17)$ & $0.0212(4)$ & \\
\hline C6 & 0.27629 (19) & $0.09175(18)$ & $0.49678(18)$ & $0.0224(4)$ & \\
\hline $\mathrm{C} 7$ & $0.3910(2)$ & $0.1567(2)$ & $0.4765(2)$ & $0.0302(5)$ & \\
\hline $\mathrm{H} 7$ & 0.3908 & 0.1983 & 0.4098 & $0.036 *$ & \\
\hline $\mathrm{C} 8$ & $0.5094(2)$ & $0.1622(2)$ & $0.5540(2)$ & $0.0358(6)$ & \\
\hline H8 & 0.5881 & 0.2080 & 0.5391 & $0.043^{*}$ & \\
\hline $\mathrm{C} 9$ & $0.5123(2)$ & $0.1027(2)$ & $0.6499(2)$ & $0.0340(5)$ & \\
\hline H9 & 0.5928 & 0.1075 & 0.7012 & $0.041 *$ & \\
\hline $\mathrm{C} 10$ & $0.1447(2)$ & 0.00486 (19) & $0.31787(17)$ & $0.0254(4)$ & \\
\hline $\mathrm{H} 10 \mathrm{~A}$ & 0.2263 & 0.0272 & 0.2886 & $0.030^{*}$ & \\
\hline H10B & 0.0709 & 0.0142 & 0.2578 & $0.030^{*}$ & \\
\hline C11 & $0.1257(2)$ & -0.11934 (19) & $0.34988(18)$ & $0.0248(4)$ & \\
\hline H11A & 0.1214 & -0.1698 & 0.2831 & $0.030 *$ & \\
\hline H11B & 0.2010 & -0.1288 & 0.4083 & $0.030 *$ & \\
\hline C12 & $-0.1075(2)$ & $-0.1898(2)$ & $0.29630(19)$ & $0.0314(5)$ & \\
\hline $\mathrm{H} 12 \mathrm{~A}$ & -0.0959 & -0.2577 & 0.2559 & $0.047 *$ & \\
\hline H12B & -0.1096 & -0.1262 & 0.2454 & $0.047 *$ & \\
\hline $\mathrm{H} 12 \mathrm{C}$ & -0.1897 & -0.2090 & 0.3233 & $0.047^{*}$ & \\
\hline C13 & $0.0092(2)$ & $-0.25829(19)$ & 0.45980 (19) & $0.0298(5)$ & \\
\hline $\mathrm{H} 13 \mathrm{~A}$ & 0.0202 & -0.3208 & 0.4115 & $0.045^{*}$ & \\
\hline
\end{tabular}




\begin{tabular}{|c|c|c|c|c|c|}
\hline H13B & -0.0719 & -0.2827 & 0.4879 & $0.045^{*}$ & \\
\hline $\mathrm{H} 13 \mathrm{C}$ & 0.0834 & -0.2401 & 0.5237 & $0.045^{*}$ & \\
\hline C14 & $0.4792(2)$ & 0.57059 (19) & $0.24055(17)$ & $0.0241(4)$ & \\
\hline H14 & 0.4000 & 0.5922 & 0.2081 & $0.029 *$ & \\
\hline $\mathrm{C} 15$ & $0.5159(2)$ & $0.5735(2)$ & $0.35907(18)$ & $0.0279(5)$ & \\
\hline $\mathrm{H} 15$ & 0.4634 & 0.5988 & 0.4049 & $0.034^{*}$ & \\
\hline C16 & $0.6272(2)$ & $0.5397(2)$ & $0.40723(18)$ & 0.0285 & \\
\hline H16 & 0.6525 & 0.5411 & 0.4870 & $0.034 *$ & \\
\hline $\mathrm{C} 17$ & $0.7048(2)$ & $0.50270(19)$ & $0.33842(17)$ & $0.0239(4)$ & \\
\hline C18 & 0.66485 (19) & $0.50777(17)$ & $0.21999(16)$ & $0.0204(4)$ & \\
\hline C19 & $0.7417(2)$ & $0.47741(18)$ & $0.14687(17)$ & 0.0215 & \\
\hline $\mathrm{C} 20$ & $0.8527(2)$ & 0.43944 (19) & $0.18998(18)$ & $0.0262(5)$ & \\
\hline $\mathrm{H} 20$ & 0.9051 & 0.4199 & 0.1409 & $0.031 *$ & \\
\hline $\mathrm{C} 21$ & $0.8896(2)$ & $0.4292(2)$ & 0.30789 (19) & $0.0285(5)$ & \\
\hline $\mathrm{H} 21$ & 0.9649 & 0.4001 & 0.3369 & $0.034^{*}$ & \\
\hline $\mathrm{C} 22$ & $0.8183(2)$ & $0.4607(2)$ & $0.37999(18)$ & $0.0268(5)$ & \\
\hline $\mathrm{H} 22$ & 0.8451 & 0.4544 & 0.4588 & $0.032 *$ & \\
\hline $\mathrm{C} 23$ & $0.2283(2)$ & 0.4009 (2) & $0.01160(18)$ & 0.0269 & \\
\hline $\mathrm{H} 23 \mathrm{~A}$ & 0.1344 & 0.3883 & -0.0234 & $0.032 *$ & \\
\hline $\mathrm{H} 23 \mathrm{~B}$ & 0.2369 & 0.4071 & 0.0944 & $0.032 *$ & \\
\hline $\mathrm{C} 24$ & $0.2821(2)$ & $0.2997(2)$ & -0.02055 (19) & $0.0278(5)$ & \\
\hline $\mathrm{H} 24 \mathrm{~A}$ & 0.2325 & 0.2276 & 0.0047 & $0.033^{*}$ & \\
\hline H24B & 0.2705 & 0.2922 & -0.1036 & $0.033^{*}$ & \\
\hline $\mathrm{C} 25$ & $0.4344(2)$ & $0.2817(2)$ & $0.15013(18)$ & $0.0282(5)$ & \\
\hline $\mathrm{H} 25 \mathrm{~A}$ & 0.3904 & 0.2006 & 0.1522 & $0.042 *$ & \\
\hline $\mathrm{H} 25 \mathrm{~B}$ & 0.3936 & 0.3307 & 0.1910 & $0.042 *$ & \\
\hline $\mathrm{H} 25 \mathrm{C}$ & 0.5269 & 0.2920 & 0.1856 & $0.042 *$ & \\
\hline $\mathrm{C} 26$ & $0.4790(2)$ & $0.2333(2)$ & $-0.0273(2)$ & $0.0321(5)$ & \\
\hline $\mathrm{H} 26 \mathrm{~A}$ & 0.5716 & 0.2419 & 0.0070 & $0.048^{*}$ & \\
\hline $\mathrm{H} 26 \mathrm{~B}$ & 0.4702 & 0.2500 & -0.1072 & $0.048 *$ & \\
\hline $\mathrm{H} 26 \mathrm{C}$ & 0.4323 & 0.1539 & -0.0203 & $0.048^{*}$ & \\
\hline $\mathrm{Cl1}$ & $0.17945(7)$ & $0.69447(6)$ & $0.09796(5)$ & $0.04344(16)$ & \\
\hline $\mathrm{Cl} 2$ & $0.17828(6)$ & $0.32313(5)$ & $0.30115(5)$ & $0.03642(14)$ & \\
\hline N1 & $0.15965(16)$ & $-0.03312(15)$ & $0.61464(14)$ & $0.0205(3)$ & \\
\hline N2 & $0.15207(16)$ & $0.08100(15)$ & $0.41938(14)$ & $0.0203(3)$ & \\
\hline $\mathrm{H} 2 \mathrm{~A}$ & 0.1392 & 0.1590 & 0.3958 & $0.024^{*}$ & \\
\hline N3 & $0.00298(16)$ & $-0.15441(15)$ & $0.39384(14)$ & $0.0216(4)$ & \\
\hline N4 & $0.54994(16)$ & $0.53923(15)$ & $0.17267(14)$ & $0.0211(4)$ & \\
\hline N5 & $0.30146(16)$ & $0.51031(16)$ & $-0.02755(14)$ & $0.0220(4)$ & \\
\hline H5 & 0.2917 & 0.5784 & 0.0167 & $0.026^{*}$ & \\
\hline N6 & $0.42293(17)$ & $0.31473(15)$ & $0.03107(14)$ & 0.0235 & \\
\hline Nil & 0.0000 & 0.0000 & 0.5000 & $0.01664(9)$ & \\
\hline $\mathrm{Ni} 2$ & 0.5000 & 0.5000 & 0.0000 & $0.01794(9)$ & \\
\hline $\mathrm{O} 1$ & $0.0938(4)$ & $0.0324(3)$ & 0.9528 & $0.0467(9)$ & 0.5 \\
\hline $\mathrm{H} 1 \mathrm{O} 1$ & $0.059(6)$ & $-0.0378(15)$ & $0.943(6)$ & $0.070 *$ & 0.5 \\
\hline $\mathrm{H} 2 \mathrm{O} 1$ & $0.055(6)$ & $0.065(4)$ & $0.992(5)$ & $0.070^{*}$ & 0.5 \\
\hline $\mathrm{O} 2$ & 0.0033 & $0.8165(2)$ & $0.91596(19)$ & $0.0614(6)$ & \\
\hline $\mathrm{H} 1 \mathrm{O} 2$ & $-0.038(4)$ & 0.778 & $0.8572(18)$ & $0.092 *$ & \\
\hline
\end{tabular}




\begin{tabular}{lllll}
$\mathrm{H} 2 \mathrm{O} 2$ & $0.034(4)$ & $0.776(3)$ & $0.966(2)$ & $0.092^{*}$ \\
$\mathrm{O} 3$ & $0.78812(19)$ & $0.40276(17)$ & $0.65477(15)$ & $0.0400(4)$ \\
$\mathrm{H} 1 \mathrm{O} 3$ & $0.794(3)$ & $0.4737(10)$ & $0.670(2)$ & $0.060^{*}$ \\
$\mathrm{H} 2 \mathrm{O} 3$ & $0.804(3)$ & $0.374(2)$ & $0.7181(14)$ & $0.060^{*}$ \\
$\mathrm{O} 4$ & $0.2998(2)$ & $0.9624(2)$ & $0.0870(2)$ & $0.0639(6)$ \\
$\mathrm{H} 1 \mathrm{O} 4$ & $0.238(3)$ & $0.987(3)$ & $0.046(3)$ & $0.096^{*}$ \\
$\mathrm{H} 2 \mathrm{O} 4$ & $0.274(4)$ & $0.8898(11)$ & $0.093(4)$ & $0.096^{*}$ \\
\hline
\end{tabular}

Atomic displacement parameters $\left(\AA^{2}\right)$

\begin{tabular}{|c|c|c|c|c|c|c|}
\hline & $U^{11}$ & $U^{22}$ & $U^{33}$ & $U^{12}$ & $U^{13}$ & $U^{23}$ \\
\hline $\mathrm{C} 1$ & 0.0267 (11) & $0.0283(12)$ & $0.0244(10)$ & $0.0095(9)$ & $0.0033(9)$ & $0.0018(9)$ \\
\hline $\mathrm{C} 2$ & $0.0372(13)$ & $0.0379(14)$ & $0.0260(11)$ & $0.0161(11)$ & $-0.0010(10)$ & $0.0061(10)$ \\
\hline $\mathrm{C} 3$ & $0.0295(12)$ & $0.0382(14)$ & $0.0310(12)$ & $0.0154(10)$ & $-0.0074(10)$ & $-0.0024(10)$ \\
\hline $\mathrm{C} 4$ & $0.0236(10)$ & $0.0261(12)$ & $0.0328(11)$ & $0.0112(9)$ & $-0.0018(9)$ & $-0.0048(9)$ \\
\hline $\mathrm{C} 5$ & $0.0179(9)$ & $0.0204(10)$ & $0.0250(10)$ & 0.0067 (8) & $0.0009(8)$ & $-0.0034(8)$ \\
\hline C6 & $0.0195(10)$ & $0.0204(10)$ & $0.0281(10)$ & $0.0074(8)$ & $0.0033(8)$ & $-0.0003(8)$ \\
\hline $\mathrm{C} 7$ & $0.0246(11)$ & $0.0251(12)$ & $0.0426(13)$ & $0.0062(9)$ & $0.0097(10)$ & $0.0040(10)$ \\
\hline $\mathrm{C} 8$ & $0.0190(10)$ & $0.0296(13)$ & $0.0577(16)$ & $0.0031(9)$ & $0.0074(10)$ & $-0.0013(11)$ \\
\hline C9 & $0.0198(10)$ & $0.0329(13)$ & $0.0470(14)$ & 0.0097 (9) & $-0.0028(10)$ & $-0.0054(11)$ \\
\hline $\mathrm{C} 10$ & $0.0273(11)$ & $0.0286(12)$ & $0.0221(10)$ & $0.0071(9)$ & $0.0085(8)$ & $0.0006(9)$ \\
\hline C11 & $0.0247(10)$ & $0.0276(12)$ & $0.0245(10)$ & $0.0089(9)$ & $0.0073(8)$ & $-0.0033(9)$ \\
\hline $\mathrm{C} 12$ & $0.0305(12)$ & $0.0312(13)$ & $0.0302(12)$ & $0.0068(10)$ & $0.0007(9)$ & $-0.0094(10)$ \\
\hline $\mathrm{C} 13$ & $0.0395(13)$ & 0.0204 (11) & $0.0320(12)$ & $0.0101(9)$ & $0.0095(10)$ & $-0.0006(9)$ \\
\hline C14 & $0.0229(10)$ & $0.0261(11)$ & $0.0249(10)$ & $0.0083(9)$ & $0.0058(8)$ & $0.0012(9)$ \\
\hline $\mathrm{C} 15$ & $0.0315(12)$ & $0.0333(13)$ & $0.0224(10)$ & $0.0100(10)$ & $0.0104(9)$ & $0.0000(9)$ \\
\hline $\mathrm{C} 16$ & $0.0331(12)$ & $0.0327(13)$ & $0.0198(10)$ & $0.0073(10)$ & $0.0053(9)$ & 0.0009 (9) \\
\hline $\mathrm{C} 17$ & $0.0251(10)$ & $0.0234(11)$ & $0.0220(10)$ & $0.0046(8)$ & $0.0024(8)$ & $0.0027(8)$ \\
\hline C18 & $0.0199(9)$ & $0.0187(10)$ & $0.0203(9)$ & $0.0025(8)$ & $0.0003(8)$ & $0.0015(8)$ \\
\hline C19 & $0.0225(10)$ & $0.0205(10)$ & $0.0210(10)$ & $0.0045(8)$ & $0.0032(8)$ & $0.0025(8)$ \\
\hline $\mathrm{C} 20$ & $0.0250(11)$ & $0.0266(11)$ & $0.0278(11)$ & 0.0075 (9) & 0.0049 (9) & 0.0039 (9) \\
\hline $\mathrm{C} 21$ & $0.0234(11)$ & $0.0312(12)$ & $0.0311(11)$ & 0.0105 (9) & $0.0003(9)$ & $0.0074(9)$ \\
\hline $\mathrm{C} 22$ & $0.0273(11)$ & $0.0297(12)$ & $0.0207(10)$ & 0.0057 (9) & $-0.0012(8)$ & $0.0062(9)$ \\
\hline $\mathrm{C} 23$ & $0.0221(10)$ & $0.0345(13)$ & $0.0253(10)$ & $0.0069(9)$ & $0.0065(8)$ & $0.0096(9)$ \\
\hline $\mathrm{C} 24$ & $0.0245(11)$ & $0.0268(12)$ & $0.0287(11)$ & $0.0002(9)$ & $0.0027(9)$ & $0.0039(9)$ \\
\hline $\mathrm{C} 25$ & $0.0331(12)$ & $0.0265(12)$ & $0.0268(11)$ & 0.0087 (9) & $0.0074(9)$ & $0.0078(9)$ \\
\hline $\mathrm{C} 26$ & $0.0436(14)$ & $0.0224(12)$ & $0.0353(12)$ & $0.0120(10)$ & $0.0143(11)$ & $0.0018(9)$ \\
\hline Cl1 & $0.0589(4)$ & $0.0479(4)$ & $0.0332(3)$ & $0.0311(3)$ & $0.0112(3)$ & $0.0026(3)$ \\
\hline $\mathrm{Cl} 2$ & 0.0428 (3) & $0.0371(3)$ & $0.0393(3)$ & 0.0192 (3) & $0.0201(3)$ & $0.0156(3)$ \\
\hline N1 & $0.0195(8)$ & $0.0222(9)$ & $0.0202(8)$ & 0.0077 (7) & $0.0013(7)$ & $-0.0009(7)$ \\
\hline N2 & $0.0191(8)$ & $0.0208(9)$ & $0.0216(8)$ & 0.0067 (7) & $0.0029(7)$ & $0.0015(7)$ \\
\hline N3 & $0.0221(8)$ & $0.0204(9)$ & $0.0223(8)$ & $0.0060(7)$ & $0.0031(7)$ & $-0.0016(7)$ \\
\hline N4 & $0.0222(8)$ & $0.0229(9)$ & $0.0183(8)$ & $0.0057(7)$ & $0.0032(7)$ & $0.0013(7)$ \\
\hline N5 & $0.0224(8)$ & $0.0249(9)$ & $0.0196(8)$ & 0.0075 (7) & $0.0037(7)$ & $0.0026(7)$ \\
\hline N6 & $0.0257(9)$ & $0.0212(9)$ & $0.0235(9)$ & $0.0062(7)$ & $0.0039(7)$ & $0.0035(7)$ \\
\hline Ni1 & $0.01521(17)$ & 0.01787 (19) & $0.01697(17)$ & $0.00518(13)$ & 0.00185 (13) & $0.00047(13)$ \\
\hline $\mathrm{Ni} 2$ & $0.01852(18)$ & $0.02006(19)$ & 0.01495 (17) & $0.00518(14)$ & $0.00156(13)$ & $0.00111(14)$ \\
\hline $\mathrm{O} 1$ & $0.056(2)$ & $0.028(2)$ & $0.051(2)$ & $0.0087(18)$ & $0.0000(19)$ & $-0.0011(17)$ \\
\hline
\end{tabular}




\begin{tabular}{lllllll}
$\mathrm{O} 2$ & $0.0768(16)$ & $0.0634(15)$ & $0.0489(12)$ & $0.0309(13)$ & $0.0067(11)$ & $0.0157(11)$ \\
$\mathrm{O} 3$ & $0.0440(10)$ & $0.0422(11)$ & $0.0364(9)$ & $0.0131(9)$ & $0.0102(8)$ & $0.0017(8)$ \\
$\mathrm{O} 4$ & $0.0583(14)$ & $0.0596(15)$ & $0.0636(15)$ & $0.0000(12)$ & $0.0018(11)$ & $-0.0004(12)$ \\
\hline
\end{tabular}

Geometric parameters $\left(A,{ }^{\circ}\right)$

\begin{tabular}{|c|c|c|c|}
\hline $\mathrm{C} 1-\mathrm{N} 1$ & $1.322(3)$ & $\mathrm{C} 19-\mathrm{N} 5^{\mathrm{i}}$ & $1.448(3)$ \\
\hline $\mathrm{C} 1-\mathrm{C} 2$ & $1.407(3)$ & $\mathrm{C} 20-\mathrm{C} 21$ & $1.419(3)$ \\
\hline $\mathrm{C} 1-\mathrm{H} 1$ & 0.9500 & $\mathrm{C} 20-\mathrm{H} 20$ & 0.9500 \\
\hline $\mathrm{C} 2-\mathrm{C} 3$ & $1.365(3)$ & $\mathrm{C} 21-\mathrm{C} 22$ & $1.362(3)$ \\
\hline $\mathrm{C} 2-\mathrm{H} 2$ & 0.9500 & $\mathrm{C} 21-\mathrm{H} 21$ & 0.9500 \\
\hline $\mathrm{C} 3-\mathrm{C} 4$ & $1.412(3)$ & $\mathrm{C} 22-\mathrm{H} 22$ & 0.9500 \\
\hline $\mathrm{C} 3-\mathrm{H} 3$ & 0.9500 & $\mathrm{C} 23-\mathrm{N} 5$ & $1.499(3)$ \\
\hline $\mathrm{C} 4-\mathrm{C} 9$ & $1.412(3)$ & $\mathrm{C} 23-\mathrm{C} 24$ & $1.515(3)$ \\
\hline $\mathrm{C} 4-\mathrm{C} 5$ & $1.418(3)$ & $\mathrm{C} 23-\mathrm{H} 23 \mathrm{~A}$ & 0.9900 \\
\hline $\mathrm{C} 5-\mathrm{N} 1$ & $1.378(3)$ & $\mathrm{C} 23-\mathrm{H} 23 \mathrm{~B}$ & 0.9900 \\
\hline $\mathrm{C} 5-\mathrm{C} 6$ & $1.418(3)$ & $\mathrm{C} 24-\mathrm{N} 6$ & $1.486(3)$ \\
\hline $\mathrm{C} 6-\mathrm{C} 7$ & $1.370(3)$ & $\mathrm{C} 24-\mathrm{H} 24 \mathrm{~A}$ & 0.9900 \\
\hline $\mathrm{C} 6-\mathrm{N} 2$ & $1.450(3)$ & $\mathrm{C} 24-\mathrm{H} 24 \mathrm{~B}$ & 0.9900 \\
\hline $\mathrm{C} 7-\mathrm{C} 8$ & $1.411(3)$ & $\mathrm{C} 25-\mathrm{N} 6$ & $1.486(3)$ \\
\hline $\mathrm{C} 7-\mathrm{H} 7$ & 0.9500 & $\mathrm{C} 25-\mathrm{H} 25 \mathrm{~A}$ & 0.9800 \\
\hline $\mathrm{C} 8-\mathrm{C} 9$ & $1.364(4)$ & $\mathrm{C} 25-\mathrm{H} 25 \mathrm{~B}$ & 0.9800 \\
\hline $\mathrm{C} 8-\mathrm{H} 8$ & 0.9500 & $\mathrm{C} 25-\mathrm{H} 25 \mathrm{C}$ & 0.9800 \\
\hline С9-H9 & 0.9500 & $\mathrm{C} 26-\mathrm{N} 6$ & $1.480(3)$ \\
\hline $\mathrm{C} 10-\mathrm{N} 2$ & $1.498(3)$ & $\mathrm{C} 26-\mathrm{H} 26 \mathrm{~A}$ & 0.9800 \\
\hline $\mathrm{C} 10-\mathrm{C} 11$ & $1.508(3)$ & $\mathrm{C} 26-\mathrm{H} 26 \mathrm{~B}$ & 0.9800 \\
\hline $\mathrm{C} 10-\mathrm{H} 10 \mathrm{~A}$ & 0.9900 & $\mathrm{C} 26-\mathrm{H} 26 \mathrm{C}$ & 0.9800 \\
\hline $\mathrm{C} 10-\mathrm{H} 10 \mathrm{~B}$ & 0.9900 & $\mathrm{~N} 1-\mathrm{Ni1}$ & 2.0909 (17) \\
\hline $\mathrm{C} 11-\mathrm{N} 3$ & $1.491(3)$ & $\mathrm{N} 2-\mathrm{Ni} 1$ & $2.1189(16)$ \\
\hline $\mathrm{C} 11-\mathrm{H} 11 \mathrm{~A}$ & 0.9900 & $\mathrm{~N} 2-\mathrm{H} 2 \mathrm{~A}$ & 1.0000 \\
\hline C11-H11B & 0.9900 & N3-Ni1 & $2.2374(17)$ \\
\hline $\mathrm{C} 12-\mathrm{N} 3$ & $1.485(3)$ & $\mathrm{N} 4-\mathrm{Ni} 2$ & $2.0778(16)$ \\
\hline $\mathrm{C} 12-\mathrm{H} 12 \mathrm{~A}$ & 0.9800 & $\mathrm{~N} 5-\mathrm{C} 19^{\mathrm{i}}$ & $1.448(3)$ \\
\hline $\mathrm{C} 12-\mathrm{H} 12 \mathrm{~B}$ & 0.9800 & $\mathrm{~N} 5-\mathrm{Ni} 2$ & $2.1143(17)$ \\
\hline $\mathrm{C} 12-\mathrm{H} 12 \mathrm{C}$ & 0.9800 & N5-H5 & 1.0000 \\
\hline $\mathrm{C} 13-\mathrm{N} 3$ & $1.481(3)$ & $\mathrm{N} 6-\mathrm{Ni} 2$ & $2.2397(17)$ \\
\hline C13-H13A & 0.9800 & $\mathrm{Ni} 1-\mathrm{N} 1^{\mathrm{ii}}$ & 2.0909 (17) \\
\hline C13-H13B & 0.9800 & $\mathrm{Ni} 1-\mathrm{N} 2^{\mathrm{ii}}$ & $2.1189(16)$ \\
\hline $\mathrm{C} 13-\mathrm{H} 13 \mathrm{C}$ & 0.9800 & $\mathrm{Ni} 1-\mathrm{N} 3^{\mathrm{ii}}$ & $2.2373(17)$ \\
\hline $\mathrm{C} 14-\mathrm{N} 4$ & $1.319(3)$ & $\mathrm{Ni} 2-\mathrm{N} 4^{\mathrm{i}}$ & $2.0777(16)$ \\
\hline $\mathrm{C} 14-\mathrm{C} 15$ & $1.411(3)$ & $\mathrm{Ni} 2-\mathrm{N} 5^{\mathrm{i}}$ & $2.1144(17)$ \\
\hline C14-H14 & 0.9500 & $\mathrm{Ni} 2-\mathrm{N} 6^{\mathrm{i}}$ & $2.2397(17)$ \\
\hline $\mathrm{C} 15-\mathrm{C} 16$ & $1.361(3)$ & $\mathrm{O} 1-\mathrm{H} 1 \mathrm{O} 1$ & $0.833(10)$ \\
\hline $\mathrm{C} 15-\mathrm{H} 15$ & 0.9500 & $\mathrm{O} 1-\mathrm{H} 2 \mathrm{O} 1$ & $0.836(10)$ \\
\hline $\mathrm{C} 16-\mathrm{C} 17$ & $1.411(3)$ & $\mathrm{O} 2-\mathrm{H} 1 \mathrm{O} 2$ & $0.835(10)$ \\
\hline $\mathrm{C} 16-\mathrm{H} 16$ & 0.9500 & $\mathrm{O} 2-\mathrm{H} 2 \mathrm{O} 2$ & $0.833(10)$ \\
\hline $\mathrm{C} 17-\mathrm{C} 22$ & $1.414(3)$ & $\mathrm{O} 3-\mathrm{H} 1 \mathrm{O} 3$ & $0.846(10)$ \\
\hline $\mathrm{C} 17-\mathrm{C} 18$ & $1.420(3)$ & $\mathrm{O} 3-\mathrm{H} 2 \mathrm{O} 3$ & $0.846(10)$ \\
\hline
\end{tabular}




\begin{tabular}{|c|c|c|c|}
\hline $\mathrm{C} 18-\mathrm{N} 4$ & $1.380(3)$ & $\mathrm{O} 4-\mathrm{H} 1 \mathrm{O} 4$ & $0.848(10)$ \\
\hline $\mathrm{C} 18-\mathrm{C} 19$ & $1.411(3)$ & $\mathrm{O} 4-\mathrm{H} 2 \mathrm{O} 4$ & $0.854(10)$ \\
\hline $\mathrm{C} 19-\mathrm{C} 20$ & $1.370(3)$ & & \\
\hline $\mathrm{N} 1-\mathrm{C} 1-\mathrm{C} 2$ & $123.5(2)$ & $\mathrm{H} 23 \mathrm{~A}-\mathrm{C} 23-\mathrm{H} 23 \mathrm{~B}$ & 108.2 \\
\hline $\mathrm{N} 1-\mathrm{C} 1-\mathrm{H} 1$ & 118.2 & $\mathrm{~N} 6-\mathrm{C} 24-\mathrm{C} 23$ & $111.42(18)$ \\
\hline $\mathrm{C} 2-\mathrm{C} 1-\mathrm{H} 1$ & 118.2 & $\mathrm{~N} 6-\mathrm{C} 24-\mathrm{H} 24 \mathrm{~A}$ & 109.3 \\
\hline $\mathrm{C} 3-\mathrm{C} 2-\mathrm{C} 1$ & $119.1(2)$ & $\mathrm{C} 23-\mathrm{C} 24-\mathrm{H} 24 \mathrm{~A}$ & 109.3 \\
\hline $\mathrm{C} 3-\mathrm{C} 2-\mathrm{H} 2$ & 120.5 & $\mathrm{~N} 6-\mathrm{C} 24-\mathrm{H} 24 \mathrm{~B}$ & 109.3 \\
\hline $\mathrm{C} 1-\mathrm{C} 2-\mathrm{H} 2$ & 120.5 & $\mathrm{C} 23-\mathrm{C} 24-\mathrm{H} 24 \mathrm{~B}$ & 109.3 \\
\hline $\mathrm{C} 2-\mathrm{C} 3-\mathrm{C} 4$ & $119.9(2)$ & $\mathrm{H} 24 \mathrm{~A}-\mathrm{C} 24-\mathrm{H} 24 \mathrm{~B}$ & 108.0 \\
\hline $\mathrm{C} 2-\mathrm{C} 3-\mathrm{H} 3$ & 120.1 & $\mathrm{~N} 6-\mathrm{C} 25-\mathrm{H} 25 \mathrm{~A}$ & 109.5 \\
\hline $\mathrm{C} 4-\mathrm{C} 3-\mathrm{H} 3$ & 120.1 & $\mathrm{~N} 6-\mathrm{C} 25-\mathrm{H} 25 \mathrm{~B}$ & 109.5 \\
\hline $\mathrm{C} 9-\mathrm{C} 4-\mathrm{C} 3$ & $124.0(2)$ & $\mathrm{H} 25 \mathrm{~A}-\mathrm{C} 25-\mathrm{H} 25 \mathrm{~B}$ & 109.5 \\
\hline $\mathrm{C} 9-\mathrm{C} 4-\mathrm{C} 5$ & $118.6(2)$ & $\mathrm{N} 6-\mathrm{C} 25-\mathrm{H} 25 \mathrm{C}$ & 109.5 \\
\hline $\mathrm{C} 3-\mathrm{C} 4-\mathrm{C} 5$ & $117.4(2)$ & $\mathrm{H} 25 \mathrm{~A}-\mathrm{C} 25-\mathrm{H} 25 \mathrm{C}$ & 109.5 \\
\hline $\mathrm{N} 1-\mathrm{C} 5-\mathrm{C} 6$ & $117.79(18)$ & $\mathrm{H} 25 \mathrm{~B}-\mathrm{C} 25-\mathrm{H} 25 \mathrm{C}$ & 109.5 \\
\hline $\mathrm{N} 1-\mathrm{C} 5-\mathrm{C} 4$ & $122.12(19)$ & $\mathrm{N} 6-\mathrm{C} 26-\mathrm{H} 26 \mathrm{~A}$ & 109.5 \\
\hline $\mathrm{C} 6-\mathrm{C} 5-\mathrm{C} 4$ & 120.09 (19) & $\mathrm{N} 6-\mathrm{C} 26-\mathrm{H} 26 \mathrm{~B}$ & 109.5 \\
\hline $\mathrm{C} 7-\mathrm{C} 6-\mathrm{C} 5$ & $119.51(19)$ & $\mathrm{H} 26 \mathrm{~A}-\mathrm{C} 26-\mathrm{H} 26 \mathrm{~B}$ & 109.5 \\
\hline $\mathrm{C} 7-\mathrm{C} 6-\mathrm{N} 2$ & $123.0(2)$ & $\mathrm{N} 6-\mathrm{C} 26-\mathrm{H} 26 \mathrm{C}$ & 109.5 \\
\hline $\mathrm{C} 5-\mathrm{C} 6-\mathrm{N} 2$ & $117.51(17)$ & $\mathrm{H} 26 \mathrm{~A}-\mathrm{C} 26-\mathrm{H} 26 \mathrm{C}$ & 109.5 \\
\hline $\mathrm{C} 6-\mathrm{C} 7-\mathrm{C} 8$ & $120.5(2)$ & $\mathrm{H} 26 \mathrm{~B}-\mathrm{C} 26-\mathrm{H} 26 \mathrm{C}$ & 109.5 \\
\hline $\mathrm{C} 6-\mathrm{C} 7-\mathrm{H} 7$ & 119.8 & $\mathrm{C} 1-\mathrm{N} 1-\mathrm{C} 5$ & $117.98(18)$ \\
\hline $\mathrm{C} 8-\mathrm{C} 7-\mathrm{H} 7$ & 119.8 & $\mathrm{C} 1-\mathrm{N} 1-\mathrm{Ni} 1$ & $128.92(14)$ \\
\hline $\mathrm{C} 9-\mathrm{C} 8-\mathrm{C} 7$ & $120.8(2)$ & $\mathrm{C} 5-\mathrm{N} 1-\mathrm{Ni} 1$ & $112.58(13)$ \\
\hline $\mathrm{C} 9-\mathrm{C} 8-\mathrm{H} 8$ & 119.6 & $\mathrm{C} 6-\mathrm{N} 2-\mathrm{C} 10$ & $110.88(16)$ \\
\hline $\mathrm{C} 7-\mathrm{C} 8-\mathrm{H} 8$ & 119.6 & $\mathrm{C} 6-\mathrm{N} 2-\mathrm{Ni} 1$ & $109.03(12)$ \\
\hline $\mathrm{C} 8-\mathrm{C} 9-\mathrm{C} 4$ & $120.5(2)$ & $\mathrm{C} 10-\mathrm{N} 2-\mathrm{Ni} 1$ & $106.62(12)$ \\
\hline $\mathrm{C} 8-\mathrm{C} 9-\mathrm{H} 9$ & 119.7 & $\mathrm{C} 6-\mathrm{N} 2-\mathrm{H} 2 \mathrm{~A}$ & 110.1 \\
\hline $\mathrm{C} 4-\mathrm{C} 9-\mathrm{H} 9$ & 119.7 & $\mathrm{C} 10-\mathrm{N} 2-\mathrm{H} 2 \mathrm{~A}$ & 110.1 \\
\hline $\mathrm{N} 2-\mathrm{C} 10-\mathrm{C} 11$ & $109.39(17)$ & $\mathrm{Ni} 1-\mathrm{N} 2-\mathrm{H} 2 \mathrm{~A}$ & 110.1 \\
\hline $\mathrm{N} 2-\mathrm{C} 10-\mathrm{H} 10 \mathrm{~A}$ & 109.8 & $\mathrm{C} 13-\mathrm{N} 3-\mathrm{C} 12$ & $106.35(17)$ \\
\hline $\mathrm{C} 11-\mathrm{C} 10-\mathrm{H} 10 \mathrm{~A}$ & 109.8 & $\mathrm{C} 13-\mathrm{N} 3-\mathrm{C} 11$ & $108.99(16)$ \\
\hline $\mathrm{N} 2-\mathrm{C} 10-\mathrm{H} 10 \mathrm{~B}$ & 109.8 & $\mathrm{C} 12-\mathrm{N} 3-\mathrm{C} 11$ & $108.26(16)$ \\
\hline $\mathrm{C} 11-\mathrm{C} 10-\mathrm{H} 10 \mathrm{~B}$ & 109.8 & $\mathrm{C} 13-\mathrm{N} 3-\mathrm{Ni1}$ & $112.67(13)$ \\
\hline $\mathrm{H} 10 \mathrm{~A}-\mathrm{C} 10-\mathrm{H} 10 \mathrm{~B}$ & 108.2 & $\mathrm{C} 12-\mathrm{N} 3-\mathrm{Ni} 1$ & $116.48(13)$ \\
\hline $\mathrm{N} 3-\mathrm{C} 11-\mathrm{C} 10$ & $110.47(17)$ & $\mathrm{C} 11-\mathrm{N} 3-\mathrm{Ni} 1$ & $103.85(12)$ \\
\hline $\mathrm{N} 3-\mathrm{C} 11-\mathrm{H} 11 \mathrm{~A}$ & 109.6 & $\mathrm{C} 14-\mathrm{N} 4-\mathrm{C} 18$ & $118.37(17)$ \\
\hline $\mathrm{C} 10-\mathrm{C} 11-\mathrm{H} 11 \mathrm{~A}$ & 109.6 & $\mathrm{C} 14-\mathrm{N} 4-\mathrm{Ni} 2$ & $129.17(14)$ \\
\hline N3-C11-H11B & 109.6 & $\mathrm{C} 18-\mathrm{N} 4-\mathrm{Ni} 2$ & $111.46(13)$ \\
\hline $\mathrm{C} 10-\mathrm{C} 11-\mathrm{H} 11 \mathrm{~B}$ & 109.6 & $\mathrm{C} 19^{\mathrm{i}-\mathrm{N}} 5-\mathrm{C} 23$ & $111.93(16)$ \\
\hline $\mathrm{H} 11 \mathrm{~A}-\mathrm{C} 11-\mathrm{H} 11 \mathrm{~B}$ & 108.1 & $\mathrm{C} 19 \mathrm{i}-\mathrm{N} 5-\mathrm{Ni} 2$ & $107.77(12)$ \\
\hline $\mathrm{N} 3-\mathrm{C} 12-\mathrm{H} 12 \mathrm{~A}$ & 109.5 & $\mathrm{C} 23-\mathrm{N} 5-\mathrm{Ni} 2$ & $106.88(12)$ \\
\hline N3-C12-H12B & 109.5 & $\mathrm{C} 19^{\mathrm{i}-\mathrm{N} 5-\mathrm{H} 5}$ & 110.1 \\
\hline $\mathrm{H} 12 \mathrm{~A}-\mathrm{C} 12-\mathrm{H} 12 \mathrm{~B}$ & 109.5 & $\mathrm{C} 23-\mathrm{N} 5-\mathrm{H} 5$ & 110.1 \\
\hline $\mathrm{N} 3-\mathrm{C} 12-\mathrm{H} 12 \mathrm{C}$ & 109.5 & $\mathrm{Ni} 2-\mathrm{N} 5-\mathrm{H} 5$ & 110.1 \\
\hline $\mathrm{H} 12 \mathrm{~A}-\mathrm{C} 12-\mathrm{H} 12 \mathrm{C}$ & 109.5 & $\mathrm{C} 26-\mathrm{N} 6-\mathrm{C} 25$ & $106.69(17)$ \\
\hline
\end{tabular}




\begin{tabular}{|c|c|c|c|}
\hline $\mathrm{H} 12 \mathrm{~B}-\mathrm{C} 12-\mathrm{H} 12 \mathrm{C}$ & 109.5 & $\mathrm{C} 26-\mathrm{N} 6-\mathrm{C} 24$ & $108.98(17)$ \\
\hline N3-C13-H13A & 109.5 & $\mathrm{C} 25-\mathrm{N} 6-\mathrm{C} 24$ & $108.49(16)$ \\
\hline $\mathrm{N} 3-\mathrm{C} 13-\mathrm{H} 13 \mathrm{~B}$ & 109.5 & $\mathrm{C} 26-\mathrm{N} 6-\mathrm{Ni} 2$ & $113.21(13)$ \\
\hline $\mathrm{H} 13 \mathrm{~A}-\mathrm{C} 13-\mathrm{H} 13 \mathrm{~B}$ & 109.5 & $\mathrm{C} 25-\mathrm{N} 6-\mathrm{Ni} 2$ & $117.61(13)$ \\
\hline $\mathrm{N} 3-\mathrm{C} 13-\mathrm{H} 13 \mathrm{C}$ & 109.5 & $\mathrm{C} 24-\mathrm{N} 6-\mathrm{Ni} 2$ & $101.49(13)$ \\
\hline $\mathrm{H} 13 \mathrm{~A}-\mathrm{C} 13-\mathrm{H} 13 \mathrm{C}$ & 109.5 & $\mathrm{~N} 1{ }^{\mathrm{ii}}-\mathrm{Ni} 1-\mathrm{N} 1$ & $180.00(9)$ \\
\hline $\mathrm{H} 13 \mathrm{~B}-\mathrm{C} 13-\mathrm{H} 13 \mathrm{C}$ & 109.5 & $\mathrm{~N} 1{ }^{\mathrm{ii}}-\mathrm{Ni} 1-\mathrm{N} 2$ & $99.10(6)$ \\
\hline $\mathrm{N} 4-\mathrm{C} 14-\mathrm{C} 15$ & $123.0(2)$ & $\mathrm{N} 1-\mathrm{Ni1}-\mathrm{N} 2$ & $80.90(6)$ \\
\hline $\mathrm{N} 4-\mathrm{C} 14-\mathrm{H} 14$ & 118.5 & $\mathrm{~N} 1^{\mathrm{ii}}-\mathrm{Ni} 1-\mathrm{N} 2^{\mathrm{ii}}$ & $80.90(6)$ \\
\hline $\mathrm{C} 15-\mathrm{C} 14-\mathrm{H} 14$ & 118.5 & $\mathrm{~N} 1-\mathrm{Ni} 1-\mathrm{N} 2^{\mathrm{ii}}$ & $99.11(6)$ \\
\hline $\mathrm{C} 16-\mathrm{C} 15-\mathrm{C} 14$ & $119.5(2)$ & $\mathrm{N} 2-\mathrm{Ni} 1-\mathrm{N} 2^{\mathrm{ii}}$ & $180.00(7)$ \\
\hline $\mathrm{C} 16-\mathrm{C} 15-\mathrm{H} 15$ & 120.3 & $\mathrm{~N} 1^{\mathrm{ii}}-\mathrm{Ni} 1-\mathrm{N} 3^{\mathrm{ii}}$ & $88.88(6)$ \\
\hline $\mathrm{C} 14-\mathrm{C} 15-\mathrm{H} 15$ & 120.3 & $\mathrm{~N} 1-\mathrm{Ni} 1-\mathrm{N} 3^{\mathrm{ii}}$ & $91.12(6)$ \\
\hline $\mathrm{C} 15-\mathrm{C} 16-\mathrm{C} 17$ & $119.85(19)$ & $\mathrm{N} 2-\mathrm{Ni} 1-\mathrm{N} 3^{\mathrm{ii}}$ & $97.02(6)$ \\
\hline $\mathrm{C} 15-\mathrm{C} 16-\mathrm{H} 16$ & 120.1 & $\mathrm{~N} 2^{\mathrm{ii}}-\mathrm{Ni} 1-\mathrm{N} 3^{\mathrm{ii}}$ & $82.98(6)$ \\
\hline $\mathrm{C} 17-\mathrm{C} 16-\mathrm{H} 16$ & 120.1 & $\mathrm{~N} 1{ }^{\mathrm{ii}}-\mathrm{Ni} 1-\mathrm{N} 3$ & $91.12(6)$ \\
\hline $\mathrm{C} 16-\mathrm{C} 17-\mathrm{C} 22$ & $124.18(19)$ & $\mathrm{N} 1-\mathrm{Ni1}-\mathrm{N} 3$ & $88.88(6)$ \\
\hline $\mathrm{C} 16-\mathrm{C} 17-\mathrm{C} 18$ & $117.32(19)$ & $\mathrm{N} 2-\mathrm{Ni} 1-\mathrm{N} 3$ & $82.98(6)$ \\
\hline $\mathrm{C} 22-\mathrm{C} 17-\mathrm{C} 18$ & $118.50(19)$ & $\mathrm{N} 2{ }^{\mathrm{ii}}-\mathrm{Ni} 1-\mathrm{N} 3$ & $97.02(6)$ \\
\hline N4-C18-C19 & $118.02(17)$ & $\mathrm{N} 3{ }^{\mathrm{ii}}-\mathrm{Ni} 1-\mathrm{N} 3$ & 180.0 \\
\hline $\mathrm{N} 4-\mathrm{C} 18-\mathrm{C} 17$ & $121.87(18)$ & $\mathrm{N} 4 \mathrm{i}-\mathrm{Ni} 2-\mathrm{N} 4$ & 180.0 \\
\hline $\mathrm{C} 19-\mathrm{C} 18-\mathrm{C} 17$ & $120.10(19)$ & $\mathrm{N} 4 \mathrm{i}-\mathrm{Ni} 2-\mathrm{N} 5$ & $81.04(6)$ \\
\hline $\mathrm{C} 20-\mathrm{C} 19-\mathrm{C} 18$ & $119.92(19)$ & $\mathrm{N} 4-\mathrm{Ni} 2-\mathrm{N} 5$ & $98.96(6)$ \\
\hline $\mathrm{C} 20-\mathrm{C} 19-\mathrm{N} 5^{\mathrm{i}}$ & $122.84(18)$ & $\mathrm{N} 44^{\mathrm{i}}-\mathrm{Ni} 2-\mathrm{N} 5^{\mathrm{i}}$ & $98.96(6)$ \\
\hline $\mathrm{C} 18-\mathrm{C} 19-\mathrm{N} 5^{\mathrm{i}}$ & $117.24(18)$ & $\mathrm{N} 4-\mathrm{Ni} 2-\mathrm{N} 5^{\mathrm{i}}$ & $81.04(6)$ \\
\hline $\mathrm{C} 19-\mathrm{C} 20-\mathrm{C} 21$ & $120.1(2)$ & $\mathrm{N} 5-\mathrm{Ni} 2-\mathrm{N} 5^{\mathrm{i}}$ & 180.0 \\
\hline $\mathrm{C} 19-\mathrm{C} 20-\mathrm{H} 20$ & 120.0 & $\mathrm{~N} 4 \mathrm{i}-\mathrm{Ni} 2-\mathrm{N} 6$ & $89.87(6)$ \\
\hline $\mathrm{C} 21-\mathrm{C} 20-\mathrm{H} 20$ & 120.0 & $\mathrm{~N} 4-\mathrm{Ni} 2-\mathrm{N} 6$ & $90.13(6)$ \\
\hline $\mathrm{C} 22-\mathrm{C} 21-\mathrm{C} 20$ & $120.8(2)$ & $\mathrm{N} 5-\mathrm{Ni} 2-\mathrm{N} 6$ & $84.17(7)$ \\
\hline $\mathrm{C} 22-\mathrm{C} 21-\mathrm{H} 21$ & 119.6 & $\mathrm{~N} 5$ - $-\mathrm{Ni} 2-\mathrm{N} 6$ & $95.83(7)$ \\
\hline $\mathrm{C} 20-\mathrm{C} 21-\mathrm{H} 21$ & 119.6 & $\mathrm{~N} 44^{\mathrm{i}}-\mathrm{Ni} 2-\mathrm{N} 6^{\mathrm{i}}$ & $90.13(6)$ \\
\hline $\mathrm{C} 21-\mathrm{C} 22-\mathrm{C} 17$ & $120.5(2)$ & $\mathrm{N} 4-\mathrm{Ni} 2-\mathrm{N} 6^{\mathrm{i}}$ & $89.87(6)$ \\
\hline $\mathrm{C} 21-\mathrm{C} 22-\mathrm{H} 22$ & 119.7 & $\mathrm{~N} 5-\mathrm{Ni} 2-\mathrm{N} 6^{\mathrm{i}}$ & $95.83(7)$ \\
\hline $\mathrm{C} 17-\mathrm{C} 22-\mathrm{H} 22$ & 119.7 & $\mathrm{~N} 5^{\mathrm{i}}-\mathrm{Ni} 2-\mathrm{N} 6^{\mathrm{i}}$ & $84.17(7)$ \\
\hline N5-C23-C24 & $109.85(17)$ & $\mathrm{N} 6-\mathrm{Ni} 2-\mathrm{N} 6^{\mathrm{i}}$ & $180.00(4)$ \\
\hline $\mathrm{N} 5-\mathrm{C} 23-\mathrm{H} 23 \mathrm{~A}$ & 109.7 & $\mathrm{H} 1 \mathrm{O} 1-\mathrm{O} 1-\mathrm{H} 2 \mathrm{O} 1$ & $110(3)$ \\
\hline $\mathrm{C} 24-\mathrm{C} 23-\mathrm{H} 23 \mathrm{~A}$ & 109.7 & $\mathrm{H} 1 \mathrm{O} 2-\mathrm{O} 2-\mathrm{H} 2 \mathrm{O} 2$ & $113(2)$ \\
\hline $\mathrm{N} 5-\mathrm{C} 23-\mathrm{H} 23 \mathrm{~B}$ & 109.7 & $\mathrm{H} 1 \mathrm{O} 3-\mathrm{O} 3-\mathrm{H} 2 \mathrm{O} 3$ & $105(2)$ \\
\hline $\mathrm{C} 24-\mathrm{C} 23-\mathrm{H} 23 \mathrm{~B}$ & 109.7 & $\mathrm{H} 1 \mathrm{O} 4-\mathrm{O} 4-\mathrm{H} 2 \mathrm{O} 4$ & $108(2)$ \\
\hline
\end{tabular}

Symmetry codes: (i) $-x+1,-y+1,-z$; (ii) $-x,-y,-z+1$.

Hydrogen-bond geometry $\left(A,{ }^{\circ}\right)$

\begin{tabular}{lllll}
\hline$D-\mathrm{H} \cdots A$ & $D-\mathrm{H}$ & $\mathrm{H} \cdots A$ & $D \cdots A$ & $D-\mathrm{H} \cdots A$ \\
\hline $\mathrm{N} 2-\mathrm{H} 2 A \cdots \mathrm{Cl} 2$ & 1.00 & 2.27 & $3.2038(18)$ & 155 \\
$\mathrm{~N} 5-\mathrm{H} 5 \cdots \mathrm{C} 11$ & 1.00 & 2.33 & $3.2715(19)$ & 156 \\
$\mathrm{O} 1-\mathrm{H} 1 O 1 \cdots \mathrm{O} 22^{\mathrm{iii}}$ & $0.83(1)$ & $1.71(1)$ & $2.541(5)$ & $173(6)$
\end{tabular}




\section{supporting information}

$\begin{array}{lllll}\mathrm{O} 1-\mathrm{H} 2 O 1 \cdots \mathrm{O} 2^{\mathrm{iv}} & 0.84(1) & 2.05(2) & 2.863(5) & 165(6) \\ \mathrm{O} 2-\mathrm{H} 1 O 2 \cdots \mathrm{Cl}^{\mathrm{v}} & 0.84(1) & 2.33(1) & 3.162(3) & 172(4) \\ \mathrm{O} 2-\mathrm{H} 2 O 2 \cdots \mathrm{Cl}^{\mathrm{vi}} & 0.83(1) & 2.37(2) & 3.179(2) & 163(4) \\ \mathrm{O} 3-\mathrm{H} 1 O 3 \cdots \mathrm{Cl} 2^{\mathrm{vii}} & 0.85(1) & 2.38(1) & 3.222(2) & 174(3) \\ \mathrm{O} 3-\mathrm{H} 2 O 3 \cdots \mathrm{Cl} 1^{\mathrm{vii}} & 0.85(1) & 2.36(1) & 3.2008(19) & 170(3) \\ \mathrm{O} 4-\mathrm{H} 1 O 4 \cdots \mathrm{O} 1^{\mathrm{viii}} & 0.85(1) & 1.91(1) & 2.755(5) & 177(4) \\ \mathrm{O} 4-\mathrm{H} 2 O 4 \cdots \mathrm{Cl} 1 & 0.85(1) & 2.33(1) & 3.180(3) & 173(3)\end{array}$

Symmetry codes: (iii) $x, y-1, z$; (iv) $-x,-y+1,-z+2$; (v) $-x,-y+1,-z+1$; (vi) $x, y, z+1$; (vii) $-x+1,-y+1,-z+1$; (viii) $x, y+1, z-1$. 